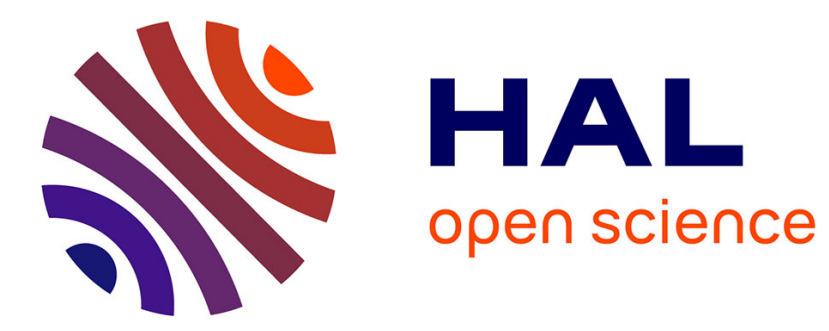

\title{
Multiplex-multiphoton microscopy and computational strategy for biomedical imaging
}

Thomas Hortholary, Claire Carrion, Emilie Chouzenoux, Jean-Christophe Pesquet, Claire Lefort

\section{- To cite this version:}

Thomas Hortholary, Claire Carrion, Emilie Chouzenoux, Jean-Christophe Pesquet, Claire Lefort. Multiplex-multiphoton microscopy and computational strategy for biomedical imaging. Microscopy Research and Technique, In press, 10.1002/jemt.23712 . hal-03126396

\section{HAL Id: hal-03126396 https://hal.science/hal-03126396}

Submitted on 31 Jan 2021

HAL is a multi-disciplinary open access archive for the deposit and dissemination of scientific research documents, whether they are published or not. The documents may come from teaching and research institutions in France or abroad, or from public or private research centers.
L'archive ouverte pluridisciplinaire HAL, est destinée au dépôt et à la diffusion de documents scientifiques de niveau recherche, publiés ou non, émanant des établissements d'enseignement et de recherche français ou étrangers, des laboratoires publics ou privés. 


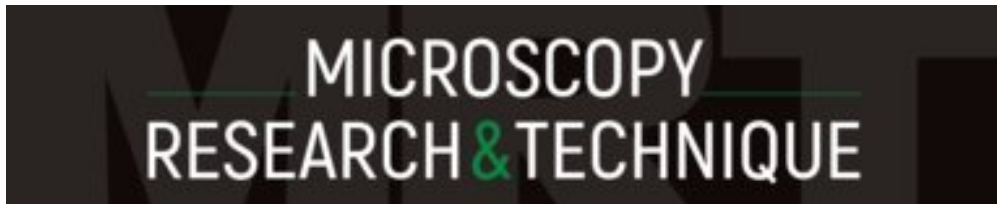

\section{Multiplex-multiphoton microscopy and computational strategy for biomedical imaging}

\begin{tabular}{|r|l|}
\hline Journal: & Microscopy Research and Technique \\
\hline Manuscript ID & MRT-20-196.R2 \\
\hline Wiley - Manuscript type: & Research Article \\
\hline Date Submitted by the & n/a \\
\hline Complete List of Authors: & $\begin{array}{l}\text { Hortholary, Thomas; UMR7252, Université de Limoges, CNRS; ENS } \\
\text { Cachan } \\
\text { Carrion, Claire; Université de Limoges, BISCEm, Microscopy core Facility } \\
\text { Chouzenoux, Emilie; CentraleSupelec; Inria Saclay-Île-de-France, Center } \\
\text { for Visual Computing } \\
\text { Pesquet, Jean-Christophe; CentraleSupelec; Inria Saclay-Île-de-France, } \\
\text { Center for Visual Computing } \\
\text { Lefort, Claire; UMR7252, Université de Limoges, CNRS }\end{array}$ \\
\hline Classifications: & $\begin{array}{l}\text { multiphoton microscopy including SHG, THG < LIGHT MICROSCOPY, } \\
\text { bioimaging < IMAGING }\end{array}$ \\
\hline Keywords: & $\begin{array}{l}\text { Multiphoton microscopy, supercontinuum lasers, PSF estimation, } \\
\text { Computational strategy, Biomedical imaging }\end{array}$ \\
\hline
\end{tabular}

\section{SCHOLARONE \\ Manuscripts}




\title{
Multiplex-multiphoton microscopy and computational strategy for biomedical imaging
}

\author{
Thomas Hortholary ${ }^{1,2}$, Claire Carrion ${ }^{3}$, Emilie Chouzenoux ${ }^{4}$, Jean-Christophe Pesquet ${ }^{4}$, Claire Lefort ${ }^{1 *}$ \\ 1. CNRS UMR 7252, XLIM Research Institute, Université de Limoges, France \\ 2. ENS Cachan, 61 avenue du Président Wilson, Cachan, France \\ 3. BISCEm, Microscopy core Facility Université de Limoges, France \\ 4. Center for Visual Computing, CentraleSupélec, INRIA Saclay, Université Paris-Saclay, France \\ *claire.lefort@xlim.fr
}

\begin{abstract}
We demonstrate the benefit of a novel laser strategy in multiphoton microscopy (MPM). The cheap, simple and turn-key supercontinuum laser system (SCLS) with its spectral shaping module, constitutes an ideal approach for the one-shot microscopic imaging of many fluorophores without modification of the excitation parameters: central wavelength, spectral bandwidth and average power. The polyvalence of the resulting multiplex-multiphoton microscopy (M-MPM) device is illustrated by images of many biomedical models from several origins (biological, medical or vegetal), generated while keeping constant the spectral parameters of excitation. The resolution of the M-MPM device is quantified by a procedure of point-spread-function (PSF) assessment led by an original, robust and reliable computational approach FIGARO. The estimated values for the PSF width for our M-MPM system are shown to be comparable to standard values found in optical microscopy. The simplification of the excitation system constitutes a significant instrumental progress in biomedical MPM, paving the way to the imaging of many fluorophores with a single shot of excitation without any modification of the lighting device.
\end{abstract}

\section{Research Highlights}

A new solution of multiplex-multiphoton microscopy device is shown, resting on a supercontinuum laser. The one-shot excitation device has imaged biomedical \& vegetal models. Our original computational strategy measures usual microscopy resolution.

\section{Introduction}

The assets of multiphoton microscopy (MPM) are now well-established in biomedical sciences, especially thanks to its near infrared range (NIR) of excitation, less dangerous and less absorbed than UV or visible excitation light (Diaspro, 2002; Mertz 2009). In routine use, standard commercial multiphoton systems include a tunable mode-locked titanium-doped sapphire laser excitation (Ti: Sa, $150 \mathrm{fs}, 80 \mathrm{MHz}, 700-1000 \mathrm{~nm}$ ) delivering a $10 \mathrm{~nm}$-spectral bandwidth at the full-width at half maximum (FWHM). This laser technology has proved its skills in MPM and has been overwhelmingly implemented on commercial MPM systems (Girkin et al., 2005, Lefort, 2018). In case of suited emission thresholds, a spectral detection is optimal for imaging many fluorophores with a single shot of excitation. Otherwise, photomultiplier tubes (PMT) are better adapted but the simultaneous detection is limited to four fluorophores at best, providing a multishot excitation strategy. Indeed, the remote multiphoton excitation spectra of standard fluorophores are spread on large bandwidths covering about $300 \mathrm{~nm}$ in the NIR, sometimes without any overlapping. This property limits the dynamic imaging of samples across different species which can only be alternatively imaged with a multishot excitation (Boppart et 
al., 2019). Then, a numerical merging of resulting images is mandatory for obtaining a multiplex image and artefacts due to misalignment during the recording can appear. This sequential procedure drastically increases imaging delay, a critical parameter in the field of dynamic multiplex imaging or for detecting phenomena at video rates (Boppart et al., 2019; Kirkpatrick et al., 2012; Abdeladim et al., 2019). The technological transition from standard multishot MPM toward a one-shot strategy of excitation for multiplex imaging thus still requires the implantation of an alternative excitation solution for MPM.

More confidential excitation solutions based on a supercontinuum laser system (SCLS) have existed in MPM for fifteen years (Palero et al., 2005, Tada et al., 2007, Li et al., 2009; Tao et al., 2011; Tu et al., 2016; Cui et al., 2017; Magnol et al., 2018; Eibl et al., 2018; Poudel et al., 2019). Such solutions are ideal for multiplexing the excitation beam thus resulting in a so called instrument of "multiplexmultiphoton microscopy" (M-MPM) system. Exogenous fluorophores such as enhanced genetically modified proteins (GFPs) have been imaged with a SCLS (Yokoyama et al. 2007; Yokoyama et al. 2008; Hou et al. 2011). But in the aforementioned works, SCLS are mostly generated by the spectral broadening of Ti: Sa pulses into a photonic crystal fiber (PCF), a strategy complex technically, expensive and not compatible with a turn-key use, mandatory for routine practice of M-MPM. Nonetheless, a large spectral bandwidth of excitation is closely associated to the question of chromatic effects. This setting is at the origin of an increase of the noise and blur level in the images. This can be detrimental to a precise quantification for the PSF instrument, though necessary for efficient restoration procedure. PSF assessment is a procedure well-established in optical microscopy which consists in: (1) imaging standardized microspheres smaller than the sought resolution, (2) numerically modeling the resulting bead images by 3D-Gaussian shapes and (3) estimating the resulting dimensions using the FWHM along the 3 dimensions. Nevertheless, available numerical solutions are often characterized by high sensitivity to noise and model restrictions (e.g. assuming zero background, or limited to 1D / 2D treatments of image projections), which may make them inefficient in the context of M-MPM PSF characterization (Zhu et al., 2013; Guo, 2011). An alternative strategy named Fourier ring correlation (FRC) provides an estimate of the FWHM of the PSF (Koho et al. 2019), resulting in a PSF model consisting in an isotropic Gaussian. This is characterized by a single FWHM value considering the main directions of the PSF aligned with the $\mathrm{X}, \mathrm{Y}$ or $\mathrm{Z}$ axis, which is not always experimentally true.

In this paper, we demonstrate the benefit of an original, cheap, simple and turn-key SCLS coupled with a spectral shaping module, devoted to biomedical MPM. The resulting M-MPM system is first dedicated to the characterization of multiphoton excitation spectra of about ten different biomedical endogenous fluorophores. Then, several models of application from life sciences are imaged with this new device and its one-shot excitation strategy. With this novel M-MPM system, no modification of the lighting device is required, regardless of the sample origin: biological, medical, chemical or vegetal. The diversity of application models imaged without technical modification of the lighting device emphasizes a significant simplification of the current routine use of MPM. The resolution associated to this new excitation strategy is quantified by PSF assessment. Our original computational approach FIGARO has been especially elaborated for M-MPM devices, likely to be confronted with deleterious chromatic effects on the effective performance of the instrument. This computational solution delivers a complete and precise 3D PSF estimation, robust to noise and anisotropic 3D blur effects which are not negligible with a wide spectral excitation (Lau et al., 2018; Chouzenoux et al., 2019). The estimated values for the PSF width for our M-MPM system are shown to be comparable to standard values found in optical microscopy. Thus, the one-shot excitation strategy with a unique spectral bandwidth removes all the needs for each sample imaged to adapt the central wavelength, spectral bandwidth and average power of laser beam. Thereby, our original M-MPM system combining a SCLS with a robust computational strategy leads to a substantial instrumental simplification in modern biomedical imaging. 
II. Multiplex-multiphoton microscopy (M-MPM) in biomedical imaging

\section{Experimental setup of M-MPM}

Figure 1 summarizes the overall proposed M-MPM optical setup combining 4 technical modules numbered from $A$ to $D$. The excitation device (Figure $1 A$ ) is composed by a SCLS which results from the spectral broadening of a monochromatic mode-locked seed laser centered at $1064 \mathrm{~nm}$ generating $5 \mathrm{ps}$ pulse durations at $40 \mathrm{MHz}$, into a photonic crystal fiber (PCF) with a $6 \mu \mathrm{m}$ core diameter. The seed laser peak power of $10 \mathrm{~kW}$ generates nonlinear processes into the PCF such as four-wave mixing, soliton effects and Raman scattering. This leads to the spectral broadening of the monochromatic spectrum from $410 \mathrm{~nm}$ up to $2.4 \mu \mathrm{m}$ with a total average power of $2 \mathrm{~W}$. At the fiber output, a parabolic mirror collimates the beam. A density filter manages the excitation average power before its injection into the spectral shaping module composed of a prism-line which plays the role of spectral selection. The resulting SCLS is spectrally and temporally characterized (Figure $1 \mathrm{~B}$ ) and then implemented on a standard configuration of upright multiphoton microscope stand (Figure 1C) from Thorlabs Inc. (Bergamo ${ }^{\circledR}$ II Series). Two PMTs with adjustable sensitivities, combined with spectral filters, detect the endogenous fluorescence emitted from the sample. The overall resolution estimation is performed by our original algorithm FIGARO for PSF assessment (Figure 1D).

The prism-line is composed of two prisms of SF57 flint dispersive glass from SCHOTT Advanced Optics, with an apex angle equal to $30^{\circ}$. An anti-reflection coating is centered at $900 \mathrm{~nm}$ on each effective surface. The first prism spatially disperses wavelengths; the second one collimates them. The distance $d$ between prisms, here equal to $4.7 \mathrm{~cm}$, and the incident angle $\vartheta$ of the beam on the first prism can be adjusted manually. This system governs the spatial spreading of wavelengths on the reflective element of the prism-line. In the current configuration proposed in Figure 1A, the back reflection mirror contains the entire spectrum spatially spread on $3.8 \mathrm{~cm}$. It is preceded by two independent slits moveable with adjustable width that can be shifted all along the spectrum. With such method, a diffraction effect is visible on the spectrum when the slits are shifted. The resolution, measured with the knife-edge method (Siegman et al., 1991), equals $4 \mathrm{~nm}$. If the excitation window required is located further in the infrared range, the moveable slits can be translated correspondingly.

\section{Spectro-temporal characteristics of excitation pulses for MPM}

The spectro-temporal characterization of excitation pulses was performed using a spectrometer (Ocean Optics, USB 2000+) and an autocorrelator (APE, Mini), positioned directly at the prism-line output (Figure 1B). Figure 2 presents the spectral and temporal characterizations of pulses measured at the prism-line output.

By increasing the distance between the two slits, central wavelength was tuned from $780 \mathrm{~nm}$ until 900 $\mathrm{nm}$ as shown in Figure 2A. For each spectral bandwidth broadened from $60 \mathrm{~nm}$ until $150 \mathrm{~nm}$ at FWHM, the corresponding autocorrelation trace was recorded and shown in Figure 2B. Figure 2C illustrates spectral bandwidth measurements at the FWHM and corresponding pulse duration as a function of the central wavelength highlighting femtosecond ranges. The NIR of excitation generated by our original excitation strategy combined with its range of pulse duration fits ideally with optimal spectrotemporal requirements of MPM. Therefore, our SCLS and its shaping module cumulate the best spectral properties for M-MPM of numerous fluorophores regardless of the biological origin of samples thanks to the cheapest, simplest and fastest managing protocol. 
Table 1. List of endogenous fluorescent substances from life sciences.

\begin{tabular}{llll}
\hline Kingdom & Samples & Molecules & Substances \\
\cline { 2 - 4 } Animals & Organs (lung, arteries, muscle...) & Proteins & Elastin, Collagen, Myosin \\
\cline { 2 - 4 } & \multirow{3}{*}{ Cells } & Proteins (enzymes) & NADH, FAD \\
\cline { 2 - 4 } & & Amino acids (in proteins) & $\begin{array}{l}\text { Tryptophan, } \\
\text { Glycine, Tyrosine... }\end{array}$ \\
\hline \multirow{3}{*}{ Plants } & Stem / leaves & Organic substances & Cellulose, Lignin, Chlorophyll \\
\cline { 2 - 4 } & Flowers / leaves & Proteins (enzymes) & NADH, FAD \\
\cline { 2 - 4 } & Cells & Amino acids (in proteins) & $\begin{array}{l}\text { Tryptophan, Phenylalanine, } \\
\text { Glycine, Tyrosine... }\end{array}$ \\
\hline
\end{tabular}

We now determine experimentally multiphoton excitation spectra of the most relevant autofluorescent substances in biomedical researches from small molecules such as amino acids to macromolecules such as proteins and organic substances. The experimental protocol proposed in (Kao et al., 2004) has been followed: each biological sample was composed of a unique extracted or synthetized solid state substance (micro-crystal or powder), placed on a microscope slice imaged with the setup presented in Figure 1. The excitation bandwidth delivered by our SCLS was fixed at $10 \mathrm{~nm}$ at FWHM and central excitation wavelength was tuned between 680 and $1000 \mathrm{~nm}$ with a step of $10 \mathrm{~nm}$ and a constant average power equal to $5 \mathrm{~mW}$ on the sample. Table 2 lists the substances tested and the spectral range of the emission filter associated. Each bandpass filter selected for this set of experiments has a spectral bandwidth of several tens of nanometers. This solution is adapted for the characterization of individual fluorophores. In a less favorable situation where several fluorophores are involved simultaneously with some overlapping emission regions, spectral detection could be favored, thus avoiding a risk of spectral crosstalk at the detection. This last solution presents however few limitations: the point-by-point spectral acquisition requires a longer acquisition duration and is less adapted in case of low signal to noise ratio emission. An intermediary solution consists in choosing emission filters detecting a peripheral part of the emission spectra, in ranges spectrally not overlapped, at the price of a weaker detected intensity and potentially degraded by noise. In the current situation, for each point of each spectrum, ten measurements were performed. The resulting excitation spectra were then normalized and averaged. Figure 3 shows the multiphoton fluorescence excitation spectra of these substances as a function of excitation wavelength.

Table 2. Multiphoton processes and detected spectral emission ranges of main endogenous fluorescent biological substances.

\begin{tabular}{lll}
\hline Substance & Multiphoton process & Detected range of fluorescence \\
\hline Elastin, Flavin (FAD), NADH & Two-photon fluorescence & $495-540 \mathrm{~nm}$ \\
\hline Glycine, Tyrosine, Collagen & Two-photon fluorescence & $420-500 \mathrm{~nm}$ \\
\hline Tryptophan & Three-photon fluorescence & $260-340 \mathrm{~nm}$ \\
\hline
\end{tabular}




\title{
4. Square and cubic dependences of multiphoton fluorescence
}

These seven biological substances highlight multiphoton fluorescence excitation spectra spread on 230 $\mathrm{nm}$, between 690 and $920 \mathrm{~nm}$. No spectral overlapping of multiphoton fluorescence excitation exists between several of these substances such as amino acids and metabolic indicators. The one-shot imaging of fluorophores presenting such a spectral gap is not possible technically with a standard Ti: Sa system having a 10-nm spectral bandwidth of excitation at the FWHM. For optimal excitation conditions, the one-shot imaging of these seven substances would require an excitation spectrum continuous on the range between 680 and $920 \mathrm{~nm}$.

\begin{abstract}
The law dependence of fluorescence intensity emitted in function with excitation average power deposed on the sample was measured for each fluorescent biological substances (Table 2). The average power was mastered with an optical density. The spectral properties remained constant during the whole experimental session: a central wavelength fixed at $900 \mathrm{~nm}$ and a spectral width of $150 \mathrm{~nm}$ at the FWHM; the excitation spectrum and the pulse duration were checked for each average power and remained not significantly modified. Figure 4 shows the logarithmic plots of the normalized fluorescence intensities measured for elastin and tryptophan in function with the logarithmic plot of the average power. The quadratic behavior is observed in Figure 4 for elastin and demonstrates the two-photon process involved; a similar quadratic evolution, not shown in Figure 4, was observed for FAD, NADH, glycine, tyrosine, and collagen. The cubic evolution of fluorescence intensity depending on average power for tryptophan demonstrates the three-photon process involved.
\end{abstract}

\section{Multiplex-multiphoton images of samples from life sciences}

In this section, we demonstrate the adaptation of our original excitation strategy to generate multiphoton images of several models of application from different fields of life sciences. The spectral properties delivered by our system remained constant during the whole experimental session and regardless of the sample imaged. Its central wavelength was fixed at $900 \mathrm{~nm}$ with a spectral width of $150 \mathrm{~nm}$ at the FWHM. The resulting excitation beam was thus implemented into our microscope stand for generating multiphoton images of different models from life sciences. Figure 5 presents the multiphoton images generated with this excitation beam. Two kinds of samples were imaged, one from the plant kingdom (douglas wood) and one from the animal kingdom (mouse kidney). The origin of the fluorescence signals was determined thanks to two information: the spectral range of emission and the spatial localization of the emitted signal on the image. For douglas wood, the endogenous fluorescence detected between 420 and $500 \mathrm{~nm}$ was located on the image from the inner part to the peripheral of the wood cell wall; the fluorescence detected between 604 and $678 \mathrm{~nm}$ was located in the peripheral region of the wood cell wall. The yellow signal represents the overlapping regions of the wood cell wall. The spatial organization presented in Figure 5A-C respectively corresponds to the lignin and cellulose localizations (Thomas et al. 2013, Donaldson et al. 2018). For the kidney sample, extracellular matrix components, flavins and lipofuscin (Zhang et al., 2018) were imaged (Figure 5D-F). Their two-photon fluorescence signals were detected respectively between 604 and $678 \mathrm{~nm}$ and between 500 and $550 \mathrm{~nm}$. The TPA spectra of these four different fluorophores ranged between 690 and $950 \mathrm{~nm}$. We notice here that no photodamage characterized by a reduction in fluorescence levels was identified all along the period of image recording. For both application model, the selection of the excitation wavelengths with the SCLS from $750 \mathrm{~nm}$ to $850 \mathrm{~nm}$ has switched off the signal emitted from the red channels (between 604 and $678 \mathrm{~nm}$ ). Reversely, the selection of the excitation wavelengths from $850 \mathrm{~nm}$ to $1000 \mathrm{~nm}$ has switched off the signal emitted from the blue channels (between 420 and $550 \mathrm{~nm}$ ). The simultaneous generation of the red and blue fluorescent signals for each application 
model was only possible thanks to a broad excitation spectrum covering a spectral range from 750 to $1000 \mathrm{~nm}$.

\section{Discussion about M-MPM in life sciences}

Figure 5 highlights the production of multiphoton fluorescent images of application models from plants and animals kingdom with a unique and unchanged excitation spectrum. Central wavelength, spectral bandwidth and average power were kept constant all along the experimental period. For the first time and regardless of the samples origin, the imaging of many fluorescent substances was achieved without any modifications on physical lighting parameters, thanks to the original and easy handled one-shot excitation strategy (Figure 1).

The multiplex and one-shot excitation strategy offered by the original M-MPM system overcomes several technical constraints associated to the standard excitation strategy of MPM. First, M-MPM is associated with the smallest time delay required to generate multiphoton signals. As described in (Diaspro et al. 2002), according to a basic principle of optics, the focal position is wavelength dependent. This phenomenon has a straightforward consequence resulting in an axial offset between focal plans recorded with excitation wavelengths spectrally pulled apart. With a standard multishot excitation, this trouble often occurs and is associated to a variable image blur between focal plans. The correction of this drawback requires a preliminary procedure of quantification which is not standard and depends on the microscope objective and the sample medium. The image blur, not always easy to detect or to correct during the recording or numerically after the image merging, results in a mismatch between positions of the same object. The SCLS, generating images all along the focal spot, allows the adjustment of focal position in live for each spectral window detected. The axial correction of the focal position become realizable before recording the image, without modification of the excitation parameters. Second, regardless of the sample origin, the one-shot excitation approach removes the constraints about central wavelength selection and wavelength dependence of average power. With a Ti: Sa laser, the wavelength adjustment is a time consuming procedure, associated to a wavelength dependent average power. The disparity in average power of a Ti: Sa laser can reach a factor 20 between its maximum at $800 \mathrm{~nm}$ and its minimum at $1000 \mathrm{~nm}$. Such variations can flawed quantitative measurements and can be harmful for sample integrity if no careful attention is paid on the average power mastering. Finally, thanks to the one-shot excitation strategy, users do not need any more to adjust the lighting device for each experiment.

The use of a single and fixed large band excitation spectrum, regardless of the biomedical model imaged from life sciences, represents a significant headway in the simplicity of use, reduced cost and dynamic performance offered by multiphoton microscopes. This instrumental simplification drives MPM towards dynamic M-MPM. This new device is associated to a new progress in biomedical imaging and in a new routine practice of microscopic characterization of biomedical models.

\section{Computational strategy for resolution assessment}

A large spectral bandwidth of excitation might be associated with chromatic effects that could be detrimental for image resolution. We quantify here the resolution performance thanks to a 3D PSF assessment procedure. Several numerical solutions of PSF estimation exist today in optical microscopy, 
Here, $\mu$ denotes the mean of the Gaussian distribution, i.e. the center of the PSF in our case, while $C$ is a $3 \times 3$ symmetric positive definite precision matrix which characterizes its width and orientation, $|\mathrm{C}|$ being its determinant. For an optimal description of the raw observed data $y$, the key point of our numerical strategy relies on a fitting of parameters $(a, b, p, \mu, C)$. This fitting task is performed using FIGARO algorithm. It is worth noting that our PSF fitting procedure does not require any pre-processing of the dataset, as it performs the denoising and fitting task jointly, assuming a Gaussian additive noise model, whose variance level is estimated automatically. The mathematical description, and numerical validation of FIGARO is detailed in our preceding publications (Lau et al., 2018; Chouzenoux et al., 2019).

Figure 6 displays the FIGARO results, starting from the image of a single microsphere cropped from native image volume. Figures $6 \mathrm{~A}$ and $6 \mathrm{~B}$ represent $2 \mathrm{D}$ projections of the acquired bead profile marginalized in XZ and XY plans, respectively. The FWHM (bleu ellipses) and center (red dots) of the PSF are computed from the $\mu$ and $C$ values estimated with FIGARO algorithm. Figures $6 C$ to $6 \mathrm{~F}$ present the FIGARO intensity profiles allowing to quantify the instrument PSF.

\section{Experimental PSF estimation}

The experimental resolution estimation of our M-MPM device is now evaluated, using our computational strategy FIGARO. We also display comparisons with the two standard PSF estimation solutions: MetroloJ and Huygens. In that purpose, yellow-green fluorescent microspheres with a 0.2 $\mu \mathrm{m}$ diameter of Invitrogen ${ }^{\mathrm{TM}}$ (reference F8811) were mounted between a microscope slide and a coverslip. Such objects serve usually as a reference standard for the calibration of the green channel of laser scanning microscopes and emit a fluorescence spectrum between 500 and $545 \mathrm{~nm}$ at the FWHM with a maximum peak at $515 \mathrm{~nm}$. With the same protocol as involved in section II.2, we have

\footnotetext{
${ }^{1}$ https://svi.nl/HuygensSoftware

2 https://imagejdocu.tudor.lu/plugin/analysis/metroloj/start

3 https://imagejdocu.tudor.lu/plugin/analysis/figaro_psf $3 \mathrm{~d}$ _optical microscopy/start
} 
determined the multiphoton excitation spectrum of these microspheres. At the FWHM, the related excitation spectrum covers the range of wavelengths between 870 and $960 \mathrm{~nm}$. The excitation beam from our SCLS were centered at $900 \mathrm{~nm}$ with a spectral bandwidth of $150 \mathrm{~nm}$ at FWHM. The 3D images were generated thanks to the XY scanning device coupled with the z-axis scan (Figure 1). The resulting 3D native image was composed of a stack of 2D squared images of $4096 \times 4096$ pixels, for a total field of view equal to $163.84 \mu \mathrm{m} \times 163.84 \mu \mathrm{m}$. The pixel size on the image equal to $0.04 \mu \mathrm{m} \times 0.04 \mu \mathrm{m}$, cumulated with a z-stack having a $0.1 \mu \mathrm{m}$ step-size on a total depth of $10 \mu \mathrm{m}$. These settings were was adjusted in order to combine an accurate acquisition of PSF image, being constituted by about ten points along each of the 3 dimensions, associated with an acceptable fitting duration of few minutes. All images were loaded in tiff format with ImageJ and 6 individual microspheres exploitable for PSF measurements were manually cropped from the native image. They were then analyzed for PSF estimation with three different computational strategies: MetroloJ, Huygens and FIGARO. Table 3 gathers the resulting PSF parameters values in the three directions of interest $\mathrm{X}, \mathrm{Y}$ and $\mathrm{Z}$.

Table 3. PSF measured from 6 microspheres in the three dimensions $X, Y$ and $Z$. Three strategies compared: MetroloJ, Huygens and FIGARO.

\begin{tabular}{c|lll|cc|c|c|cc}
\hline $\begin{array}{c}\text { Bead } \\
\text { number }\end{array}$ & \multicolumn{3}{c}{ MetroloJ } & \multicolumn{3}{c|}{ Huygens } & \multicolumn{3}{c}{ FIGARO } \\
\hline 1 & 0.004 & 0.038 & 0.006 & 0.304 & 0.310 & 7.82 & 0.300 & 0.323 & 2.275 \\
2 & 0.109 & 0.038 & 1.38 & 0.510 & 1.078 & 1.969 & 0.364 & 0.674 & 2.572 \\
3 & 0.129 & 0.136 & 1.688 & 0.328 & 0.313 & 12.564 & 0.205 & 0.249 & 2.85 \\
4 & 0.04 & 0.038 & 0.009 & 0.356 & 0.343 & 4.063 & 0.599 & 0.458 & 2.627 \\
5 & 0.007 & 0.038 & 0.007 & 0.339 & 0.340 & 6.736 & 0.459 & 0.275 & 0.969 \\
6 & 0.106 & 0.022 & 0.051 & 0.306 & 0.320 & 3.529 & 0.334 & 0.681 & 2.572 \\
\hline $\begin{array}{c}\text { Average } \\
\text { Value }\end{array}$ & $\mathbf{0 . 0 7}$ & $\mathbf{0 . 0 5}$ & $\mathbf{0 . 5}$ & $\mathbf{0 . 4}$ & $\mathbf{0 . 4}$ & $\mathbf{6 . 1}$ & $\mathbf{0 . 4}$ & $\mathbf{0 . 4}$ & $\mathbf{2 . 3}$ \\
\hline $\begin{array}{c}\text { Standard } \\
\text { deviation }\end{array}$ & $\mathbf{0 . 0 5}$ & $\mathbf{0 . 0 4}$ & $\mathbf{0 . 7}$ & $\mathbf{0 . 0 8}$ & $\mathbf{0 . 2 9}$ & $\mathbf{3 . 5}$ & $\mathbf{0 . 1 3}$ & $\mathbf{0 . 1 8}$ & $\mathbf{0 . 6}$ \\
\hline
\end{tabular}

\section{Discussion about PSF estimation and related computational strategies}

The resolution performance is an essential parameter in microscopy, quantified by a standardized practice of PSF estimation. The values, resulting to a protocol of PSF estimation, seem to highly depend on the mathematical procedure being used. Here, three different PSF estimation procedures have been compared for the evaluation of the resolution performance of our M-MPM. For each PSF estimation, the value of standard deviation has been calculated.

The standard methods of PSF assessment with "MetroloJ" and "Huygens", highlight guarded and highly dispersed results of resolution. Those values illustrate the limits of the underlying mathematical strategies in those two tools, which are facing their limits in the situation of M-MPM. Mathematically, both Huygens and MetroloJ process the 3 directions $X, Y$ and $Z$ separately, using standard curve-fitting strategy, and thus obtain only 1D characterizations of the PSF along those 3 directions. This procedure is not precise enough, and unsuitable for noise levels and highly anisotropic blur associated to M-MMP.

A relevant PSF estimation strategy must consider 3D data and deal with essential mathematical properties such as guaranties of convergence and background not assumed to be equal to zero. Our computational strategy FIGARO, especially devoted to M-MPM resolution estimation, has specifically integrated these properties into its mathematical protocol. With this strategy, the resolution estimation equals $0.4 \mu \mathrm{m}$ in the $X$ and $Y$ directions and $2.3 \mu \mathrm{m}$ in the $Z$ direction. Our computational strategy has highlighted its strong relevance for the analysis of PSF data coming from M-MPM, with a 
low dispersion, and has demonstrated a better resistance to noise and blur than standard strategies. Our FIGARO strategy allows to distinguish the signal coming specifically from microspheres to the noise generated by the large spectral bandwidth of excitation. The estimated resolution values were in adequacy with standard resolutions of optical microscopes (Diaspro et al. 2002): a planar submicrometer resolution (optimal value theoretically estimated at $361 \mathrm{~nm}$ ) and an axial micrometer resolution (optimal value theoretically estimated at $1.58 \mu \mathrm{m}$ ). Our M-MPM device thus does not suffer from chromatic aberrations more than standard optical microscopy systems, emphasizing lateral and axial resolution performance correctly preserved compared to standards usually encountered with a monochromatic excitation.

\section{Conclusion}

Multiphoton microscopy (MPM) is a technical solution well-adapted for dynamic imaging or for revealing complex biomedical structures. But the quasi-monochromatic excitation systems usually implemented in commercial multiphoton microscopes limits at four the quantity of fluorophores that can be imaged simultaneously at best or imposes a multishot imaging approach of each individual fluorophore of interest. Such a sequential procedure is time-consuming and associated with several engineering locks, likely corrupting image information. In that context, a large spectral bandwidth of excitation has gained interest. Implemented on a multiphoton microscope, this new excitation strategy results into a so called multiplex-MPM (M-MPM) system. In this paper, we have highlighted an original engineering solution of M-MPM based on a simple, cheap and reliable supercontinuum laser source coupled with a passive spectral shaping module. This one-shot excitation strategy turns out to provide a new solution with temporal performance favorable to generate multiphoton processes. The polyvalence of the resulting M-MPM system was illustrated by images of biomedical models from several origins (biological, medical or vegetal) while keeping constant the spectral parameters of excitation. To characterize the resolution performance of this new device, we have developed an original and novel computational strategy. This approach, especially devoted to M-MPM devices, rests on a rigorous mathematical approach based on the more recent and sophisticated methods of numerical estimation. A preserved optical resolution was shown compared to standard optical microscopy systems. Henceforth, many new perspectives are now opened in dynamic biomedical microscopy thanks to M-MPM associated with a robust computational strategy.

\section{Acknowledgments}

This study was supported by the French National Center for Scientific Research (CNRS) with its program AAP interne 2018 SupRéMA. The authors would like to especially thank Dr Guy Costa for his help in providing wood samples and Dr Jeanne Cook-Moreau for her careful proofreading and correction of English. 


\section{References}

(Abdeladim et al., 2019) Abdeladim L., Matho KS, Clavreul S, Mahou P, Sintes JM, Solinas X, Arganda-Carreras I, Turney SG, Lichtman JW, Chessel A, Bemelmans AP, Loulier K, Supatto W, Livet J, Beaurepaire E. 2019. Multicolor multiscale brain imaging with chromatic multiphoton serial microscopy. Nature Communication, 10.

(Boppart et al. 2019) BoppartSA, You S, Li L, Chen J, Tu H. 2019. Simultaneous label-free autofluorescence-multiharmonic microscopy and beyond. ALP Photonics, 4, 100901.

(Chouzenoux et al., 2019) Chouzenoux E, Lau TTK, Lefort C, Pesquet JC. 2019. Optimal Multivariate Gaussian Fitting with Applications to PSF Modeling in Two-Photon Microscopy Imaging. Journal of Mathematical Imaging and Vision, article in press.

(Cui et al. 2017) Cui Q, Chen Z, Liu Q, Zhang Z, Luo Q, Fu L. 2017. Visible continuum pulses based on enhanced dispersive wave generation for endogenous fluorescence imaging. Biomedical Optics Express, 8 (9), 4026 - 4036.

(Diaspro, 2002) Diaspro A. 2002. Confocal and Two-photon microscopy, Foundations, applications and advances. Wiley-Liss Inc., New York.

(Donaldson et al. 2018) L. Donaldson, N. Williams, "Imaging and Spectroscopy of Natural Fluorophores in Pine Needles", Plants, 7 (10), 1-16 (2018)

(Eibl et al., 2018) Eibl M, Weng D, Hakert H, Kolb JP, Pfeiffer T, Hundt JE, Huber R, Karpf S. 2018. Wavelength agile multiphoton microscopy with a fiber amplified diode laser. Biomedical Optics Express, 9 (12), 6273-6282.

(Girkin et al., 2005) Girkin JM, McConnell G. 2005. Advances in Laser sources for confocal and multiphoton microscopy. Microscopy research and technique, 67, 8-14.

(Guo 2011) Guo H. 2011. A simple algorithm for fitting a Gaussian function [DSP tips and tricks]. IEEE Signal Proc. Mag., 28(5), 134-137 (2011)

(Hou et al. 2011) Hou X and Cheng W, "Single-molecule detection using continuous wave excitation of two-photon fluorescence", Opt. Lett. 36 3185-7 (2011)

(Kao, 2004) Kao FJ. 2004. The Use of Optical Parametric Oscillator for Harmonic Generation and Two-Photon UV Fluorescence Microscopy. Microscopy Research and Technique, 63, 175-181.

(Kirkpatrick et al., 2012) Kirkpatrick N, Chung E, Cook D, Han X, Gruionu G, Liao S, Munn L, Padera T, Fukumura D, Jain R K. 2012. Video-rate resonant scanning multiphoton microscopy: An emerging technique for intravital imaging of the tumor microenvironment. IntraVital, 1 (1), 60-68.

(Koho et al. 2019) S. Koho, G. Tortarolo, M. Castello, T. Deguchi, A. Diaspro, G. Vicidomini, "Fourier ring correlation simplifies image restoration in fluorescence microscopy", Nature Communication 10:3103 (2019)

(Lau et al., 2018) Lau TTK, Chouzenoux E, Lefort C, Pesquet JC. 2018. Optimal Multivariate Gaussian Fitting for PSF Modeling in Two-Photon Microscopy. Proceedings of the IEEE International Symposium on Biomedical Imaging.

(Lefort, 2018) Lefort C. 2018. Laser sources in multiphoton microscopy: Overview and optimization. Proceedings of SPIE Unconventional Optical Imaging, $106770 \mathrm{~V}$.

(Lefort et al. 2016) Lefort C., O'Connor R. P., Blanquet V., Magnol L., Kano H., Tombelaine V., Lévêque P., Couderc V., Leproux P., Multicolor multiphoton microscopy based on a nanosecond supercontinuum laser source, J. Biophotonics, 9 (7) 709-714 (2016)

(Li et al., 2009) Li D, Zheng W, Qu JY. 2009. Two-photon autofluorescence microscopy of multicolor excitation. Optics Letters, 34 (2), 202-204.

(Magnol et al., 2018) Magnol L, Blanquet V, Lefort C. 2018. Physical parameters of ultrawide band laser sources and their impacts on multiphoton imaging. Proceedings of SPIE, Nonlinear Optics and its Applications, $106841 \mathrm{~B}$.

(Mertz, 2009) Mertz J. 2009. Introduction to Optical Microscopy. Roberts \& Company Publishers.

(Palero et al., 2005) Palero JA, Boer VO, Vijverberg JC, Gerritsen HC, Sterenborg HJCM. 2005. Short-wavelength two-photon excitation fluorescence microscopy of tryptophan with a photonic crystal fiber based light source. Optics Express, 13, 53635368.

(Poudel et al. 2019) Poudel C, Kaminski CF. 2019. Supercontinuum radiation in fluorescence microscopy and biomedical imaging applications. JOSA B, 36 (2), A139-A153.

(Siegman et al., 1991) Siegman AE, Sasnett MW, Johnston TF. 1991. Choice of clip levels for beam width measurements using knife-edge techniques. IEEE Journal of Quantum Electronics, 27, 4.

(Tada et al., 2007) Tada J, Kono T, Suda A, Mizuno H, Miyawaki A, Midorikawa K, Kannari F. 2007. Adaptively controlled supercontinuum pulse from a microstructure fiber for two-photon excited fluorescence microscopy. Appl. Opt. 46 3023-30. (Tao et al., 2011) Tao W, Bao H, Gu M. 2011. Enhanced two-channel nonlinear imaging by a highly polarized supercontinuum light source generated from a nonlinear photonic crystal fiber with two zero-dispersion wavelengths. J. Biomed. Opt., 16, 056010.

[Thomas et al. 2013] Jimmy Thomas, Manfred Ingerfeld, Hema Nair, Shakti Singh Chauhan, David A. Collings, "Pontamine fast scarlet 4B: a new fluorescent dye for visualising cell wall organisation in radiata

pine tracheids", Wood Sci Technol, 47, 59-75 (2013) 
(Tu et al., 2016) Tu H, Liu Y, Turchinovich D, Marjanovic M, Lyngs J, Lægsgaard J, Chaney EJ, Zhao Y, You S, Wilson LW, Xu B, Dantus M, Boppart SA. 2016. Stain-free histopathology by programmable supercontinuum pulses. Nature Photonics, 10 (8), 534-540.

(Yokoyama et al. 2007) Yokoyama, H, Tsubokawa, H, Guo, H, Shikata, J.-I., Sato, K.-I., Takashima, K., Kashiwagi, K., Saito, N., Taniguchi, H., Ito, H., "Two-photon bioimaging utilizing supercontinuum light generated by a high-peak-power picosecond semiconductor laser source", J. Biomed Optics 12(5). 054019 (2007)

(Yokoyama et al. 2008) H. Yokoyama, A. Sato, H. -C. Guo, K. Sato, M. Mure, and H. Tsubokawa, "Nonlinear-microscopy opticalpulse sources based on mode-locked semiconductor lasers", Optics Express, 16 (22) (2008)

(Zhang et al., 2018) Zhang Y, Wang Y, Cao WW, Ma KT, Ji W, Han ZW, Si JQ, Li L. 2018. Spectral Characteristics of Autofluorescence in Renal Tissue and Methods for Reducing Fluorescence Background in Confocal Laser Scanning Microscopy. Journal of Fluorescence, 28 (2), 561-572.

(Zhu et al., 2013) Zhu X, Zhang D. 2013. Efficient parallel Levenberg-Marquardt model fitting towards real-time automated parametric imaging microscopy. Plos One 8 (10), 1-9. 


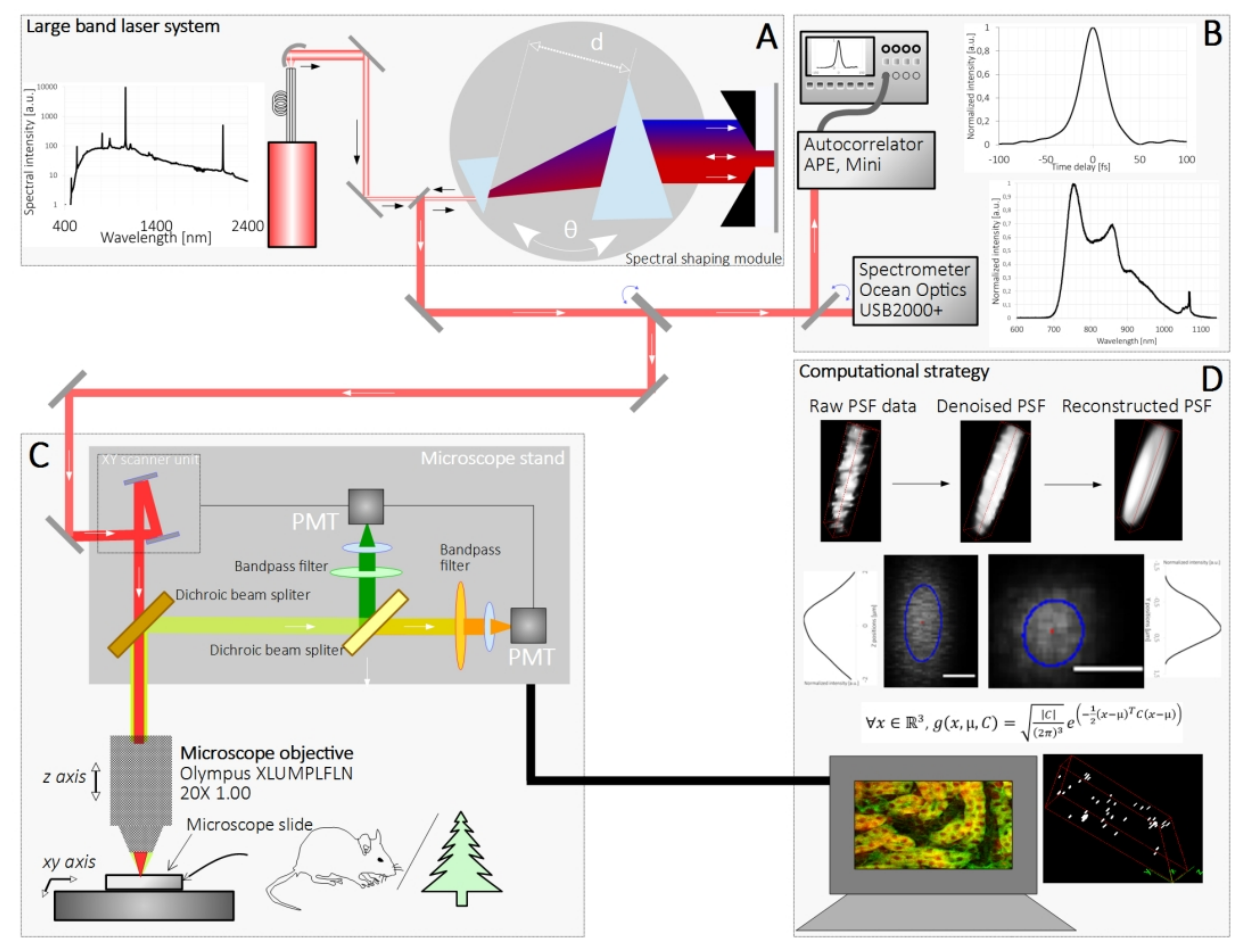

Experimental setup of the M-MPM device with a SCLS and its spectral shaping module (A, B) for multiphoton imaging of samples from life sciences (C) combined with our original computational strategy for PSF assessment (D). 


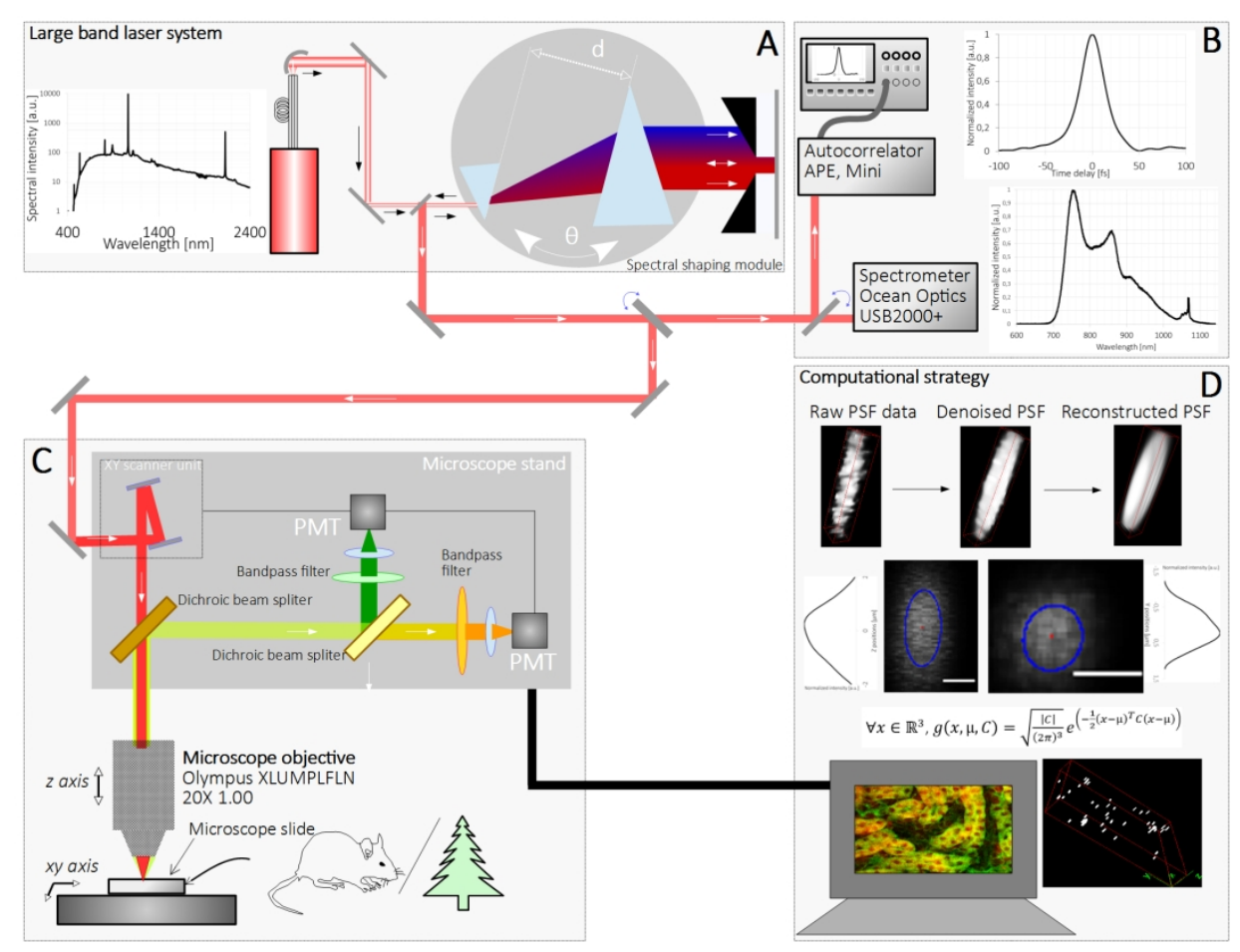

Figure 1. Experimental setup of the M-MPM device with a SCLS and its spectral shaping module $(A, B)$ for multiphoton imaging of samples from life sciences (C) combined with our original computational strategy for PSF assessment (D). 

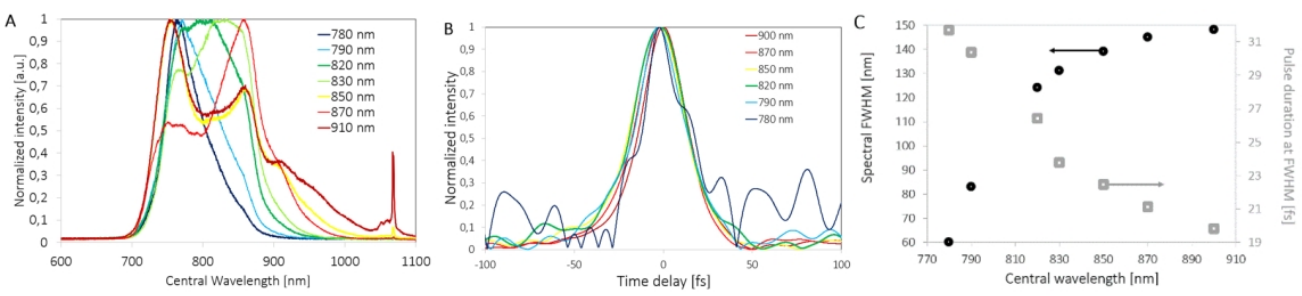

Figure 2. A. Spectral shape and B. Temporal shape of pulses central wavelengths between 780 and $900 \mathrm{~nm}$ and spectral bandwidths at FWHM between 60 and $150 \mathrm{~nm}$. C. Spectral bandwidth at the FWHM (nm) and related experimental pulse duration (fs) as a function of central wavelength $(\mathrm{nm})$. For each plot, an arrow points to the corresponding ordinate scale.

$638 \times 154 \mathrm{~mm}(72 \times 72 \mathrm{DPI})$ 


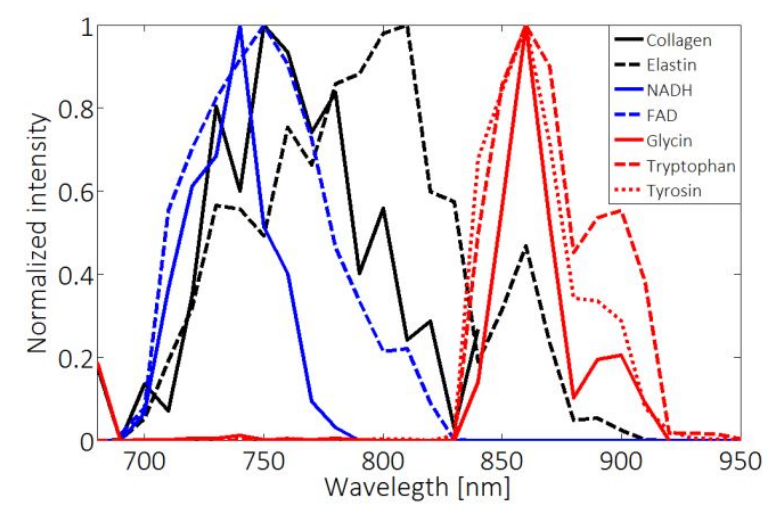

Figure 3. Experimental measurements of multiphoton fluorescence excitation spectra of endogenous fluorescent biological substances of interest in biomedical researches as a function of excitation wavelength: proteins (collagen, elastin), enzymes (NADH, FAD) and amino-acids (glycine, tyrosine and tryptophan). 


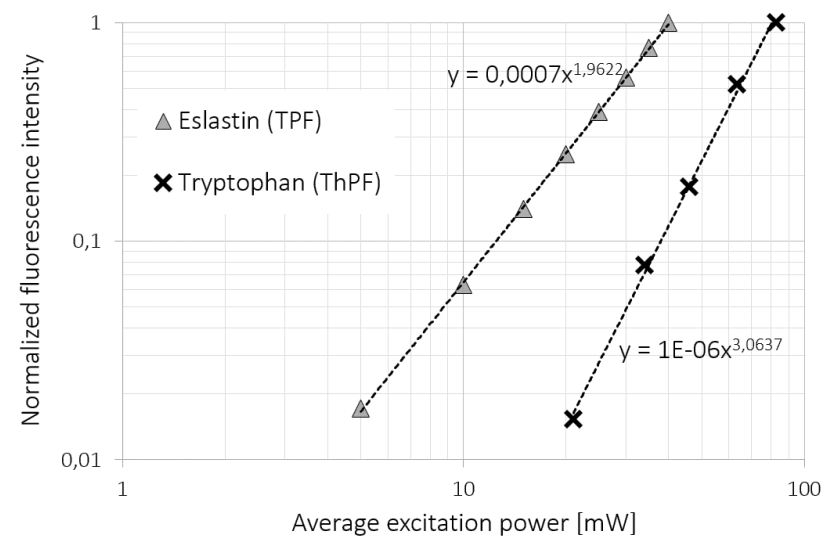

Figure 4. Normalized fluorescence intensities in function with average excitation power for elastin and tryptophan. Square and cubic dependences of multiphoton fluorescence are highlighted. 
Figure 5. Illustration of multiphoton images of several biological samples obtained with a unique large band multiphoton excitation centered at $900 \mathrm{~nm}$ with a spectral bandwidth of $150 \mathrm{~nm}$ at FWHM. Images with $2048 \times 2048$ pixels were acquired with a pixel dwell-time of $3.6 \mu \mathrm{s} / \mathrm{pixel}$. A to C: Endogenous fluorescent Douglas wood detected between 604 and $678 \mathrm{~nm}(\mathrm{~A})$, between $420 \mathrm{~nm}$ and $500 \mathrm{~nm}$ (B), and merged (C). D to F: Kidney autofluorescence detected between 604 and $678 \mathrm{~nm}$ (D), and between 500 and $550 \mathrm{~nm}$ (E), and merged (F). Scale bar: $50 \mu \mathrm{m}$. 

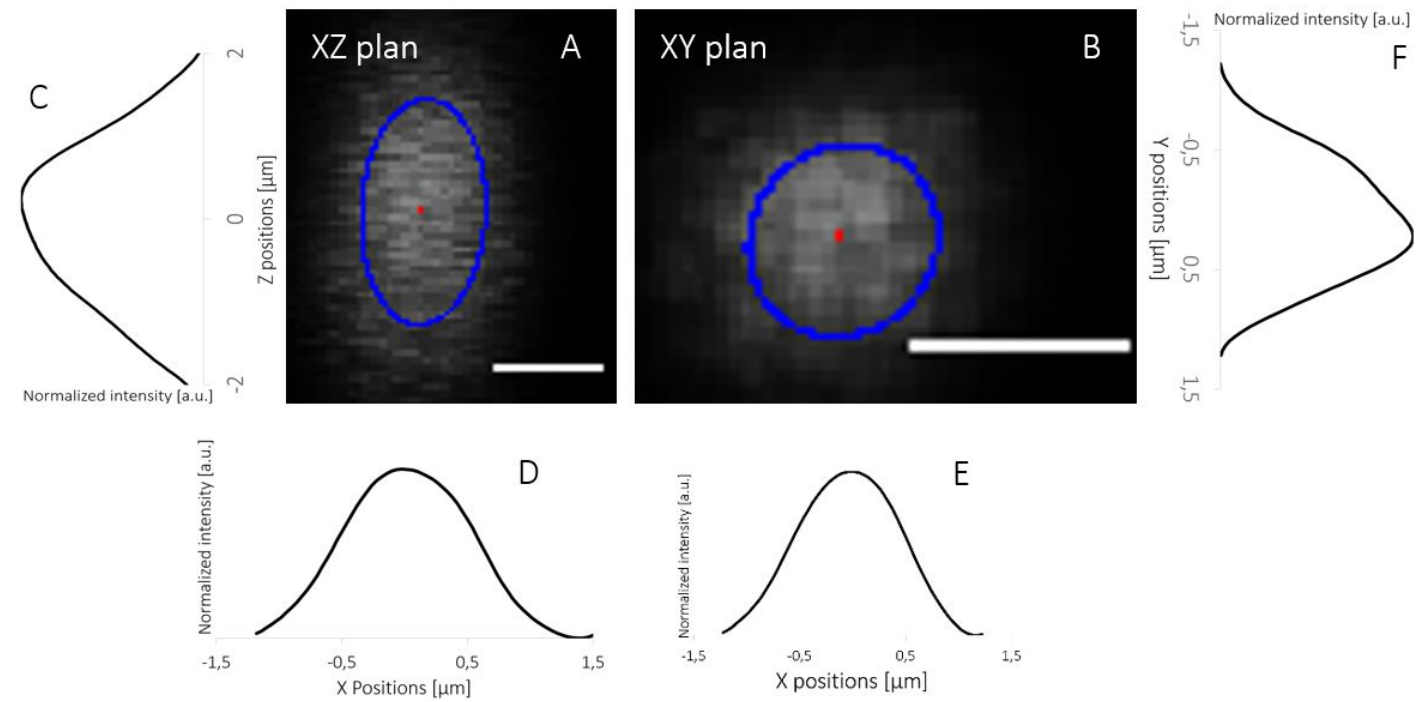

Figure 6. PSF characterization of our M-MPM device. Images of yellow-green fluorescent microspheres (diameter: $0.2 \mu \mathrm{m}$, detection range 500-550 nm); excitation centered at $900 \mathrm{~nm}, 150 \mathrm{~nm}$ at FWHM. A and B. XZ and XY 2D image of a single microsphere after estimating PSF with FIGARO plugin. Red dots and blue ellipses localize respectively the bead center and the computed FWHM of intensity with an analytical Gaussian model. C and D. PSF intensity profile in XZ plan. E and F. PSF intensity profile in XY plan. Scale bars: $1 \mu \mathrm{m}$. 